\title{
Extrapolation of Atomic Natural Orbitals of basis set to complete basis set limit
}

\author{
Jaroslav Granatier \\ Institute of Physical Chemistry and Chemical Physisc, Slovak University of Technology in Bratislava, \\ Radlinského 9, SK-812 37 Bratislava, Slovakia \\ jaroslav.granatier@stuba.sk
}

\begin{abstract}
Relativistic Atomic Natural Orbitals (ANO-RCC) are extrapolated to the complete basis set limit. ANO-RCC-VXZP $(\mathrm{X}=\mathrm{D}, \mathrm{T}, \mathrm{Q})$ basis sets were extrapolated using standard extrapolation techniques. Five noncovalent complexes, characterized by hydrogen, dispersion and halogen interactions, were chosen. Accurate description of the studied complexes is allowed only after the inclusion of electron correlation and large basis sets which have to include polarization and diffuse functions. Results are in quantitative agreement with the benchmark data obtained by standard aug-cc-pVXZ-DK $(X=\mathrm{D}, \mathrm{T}, \mathrm{Q})$ basis sets considering chemical accuracy of $\pm 1 \mathrm{kcal} / \mathrm{mol}$.
\end{abstract}

Keyword: basis sets extrapolation, ANO-RCC, aug-cc-pVXZ-DK, noncovalent interaction, MP2, CCSD(T)

\section{Introduction}

Noncovalent interactions play crucial role in many chemical systems or processes. Although noncovalent interactions exhibit considerably weaker stability compared to covalent bonds, they significantly influence the stability, structure and properties of e.g. biomolecular systems (such as DNA, RNA or proteins, Vondrášek et. al., 2005; Riley et. al., 2006; Vondrášek et. al., 2007; Berka et. al., 2009). Similarly, complexes of neutral metal atoms, such as $\mathrm{Cu}, \mathrm{Ag}, \mathrm{Au}$ or $\mathrm{Pt}$ with lone pair ligands (ligands with lone electron pair, such as ammonia, water or phosphine) or aromatic molecules (benzene, coronene) are bonded by weak noncovalent interactions (Granatier et. al., 2010; Granatier et. al., 2011; Granatier et. al., 2013). Hydrogen bonds, dispersion and charge-transfer interactions, or halogen bonds are the most prevalent types of noncovalent interactions.

An accurate description of complexes stabilized by noncovalent interactions requires the use of the most sophisticated computational methods:

- First of all, electron correlation effects, such as perturbation or coupled cluster approaches, have to be accounted for. The coupled cluster method with single and double excitations and perturbative triple correction $(\mathrm{CCSD}(\mathrm{T})$, Raghavachari et. al., 1989), represents the gold standard of computational chemistry for assessing noncovalent interactions of closed shell (single reference) species. However, the long computational time and high computational demands of this approach, scaling is $O\left(\mathrm{~N}^{7}\right)$ with the size of the basis set, allow the use of this method only for small systems. The CCSD(T) approach in combination with an extended basis set (see the point below) provides interaction energies for various types of noncovalent chemical complexes as well as higher accuracy ( \pm 1 or $\pm 0.1 \mathrm{kcal} / \mathrm{mol}$ ) (Riley et. al., 2010). A large part of electron correlation can be covered using the second order Møller-Plesset perturbation theory (MP2) [Møller et. al., 1934] which allows calculating molecular systems with the size of 100 atoms due to its lower computational demands (scaling is $O\left(\mathrm{~N}^{5}\right)$ with the size of the basis set) compared to the $\operatorname{CCSD}(\mathrm{T})$ method. Although the MP2 method describes well the most important part of the interaction energy, such as the induction, charge-transfer, electrostatic and exchange contributions, the dispersion component is largely overestimated.

- The second important factor in an accurate calculation of noncovalent complexes is the basis set quality including polarization and diffuse functions. However, correlation energy, which plays an important role in the description of noncovalent complexes, slowly converges with the size of the basis set. Consequently, the CCSD(T) correlation calculations of medium size or large noncovalent molecular complexes with very large basis sets are still impractical. Thus, design of extrapolation techniques for the correlation energy estimation in complete basis set limit is desirable.

Very useful studies on the basis set convergence and the extrapolation to the complete basis set limit suggestions were presented by Martin (Martin, 1996; Martin et. al., 1997; Martin, 1998) and Halkier (Halkier et. al., 1996). Both extrapolation schemes were used to obtain very accurate results for small molecules. However, these results were extrapolated from calculations performed in large aug-cc-pVXZ $(\mathrm{X}=\mathrm{T}, \mathrm{Q}, 5)$ or cc-pCVXZ $(\mathrm{X}=5,6)$ basis sets, 
where $\mathrm{X}$ is the cardinality number of the basis set. When considering the cc-pVDZ or cc-pCVDZ, the extrapolation accuracy decreases. Halkier et al. recommended the optimization of extrapolation exponents to minimize the differences between the best estimate of the basis set limit and the result obtained by extrapolation of basis sets with a lower cardinality number. This idea was applied also by Truhlar (Truhlar, 1998) who suggested the values of extrapolation exponents to be 3.4, 2.2 and 2.4 for HF, MP2 and CCSD(T) levels of theory from calculations performed in cc-pVDZ and cc-pVTZ basis sets, respectively.

Jurečka et al. (Jurečka et. al., 2002) showed that the difference between the $\operatorname{CCSD}(\mathrm{T})$ and MP2 interaction energies converge faster to the complete basis set limit than those calculated at the MP2 and CCSD(T) levels of theory. Thus, the MP2 interaction energy is extrapolated to the complete basis set limit and then corrected for the difference between CCSD(T) and MP2 interaction energies calculated using the smaller basis set used:

$$
\Delta E_{C B S}^{C C S D(T)}=\Delta E_{C B S}^{M P 2}+\left(\Delta E^{C C S D(T)}-\Delta E^{M P 2}\right)_{\text {small }},
$$

where $\triangle E_{C B S}^{M P 2}$ represents the extrapolated MP2/CBS interaction energy, and the difference in parentheses is the correction term estimated as the difference of the interaction energies $\Delta E^{C C S D(T)}$ and $\Delta E^{M P 2}$ calculated in a smaller basis set. At present, this approach represents the standard method for benchmark CCSD(T)/CBS interaction energies determination (Pitoňák et. al., 2008; Pitoňák et. al., 2009; Pitoňák et. al., 2010). Optimized structures calculated at the $\operatorname{CCSD}(\mathrm{T}) / \mathrm{CBS}$ level of theory as defined by eq. 1 are collected in databases such as S22, S66, X40 or A24 (Jurečka et. al., 2006; Rezáč et. al., 2011; Rezáč et. al., 2012; Řezáč et. al., 2013). These databases contain exclusively complexes bonded by weak noncovalent interactions e.g. hydrogen, dispersion or halogen bonds, respectively, and are designed for the development and benchmarking of new approaches or methods.

The presented paper is focused on the extrapolation of relativistic all-electron ANO (ANO-RCC) basis sets (Roos et. al., 2004, Roos et. al., 2005). The ANORCG basis sets include polarization and diffuse functions and hence are useful for the calculation of noncovalent interactions. Furthermore, ANO-RCC basis sets are available for the entire periodic table. Another advantage of these basis sets is that they are available with various degrees of contraction (cardinal numbers). However, these basis sets contain the same exponents for all degrees of contraction which is partially a disadvantage for the extrapolation compared to basis sets such as cc-pVXZ or aug-cc-pVXZ (Peterson, 2015). Herein, it is proved that basis sets of the ANO-type can be still used for the extrapolation of binding energies of noncovalent complexes to the complete basis set limit with chemical accuracy (or higher). Interaction energies evaluated by extrapolation of the ANO-RCC basis set are compared with results obtained by extrapolation of the standard aug-cc-pVXZ basis sets.

\section{Computation details}

Numerical results presented in this paper were obtained by the MOLCAS 8.0 quantum chemistry programs (Karlström et. al., 2003). All studied complexes (methane... $\mathrm{F}_{2}$, water...ammonia, water... dimer, HF...methanol and benzene...methane) are characterized by weak noncovalent interactions and are part of the benchmark CCSD(T)/CBS databases for new approaches design and testing. Benchmark interaction energies and the corresponding structures were taken from the website http://www.begdb.com. The benchmark interaction energies as well as the structures noted on the mentioned website were obtained by nonrelativistic calculations. However, our benchmark results were obtained at the relativistic MP2 and CCSD(T) level of theory using the aug-cc-pVXZ-DK basis sets. All studied complexes contain hydrogen and second period atoms. Therefore, with the exception of the $1 \mathrm{~s}^{2}$ electrons of the second period atoms, all electrons in the complexes were correlated. All calculations were performed relativistic basis sets. Therefore, the relativistic effects were included using the scalar relativistic Douglas-Kroll-Hess (DKH) approximation (Douglas et. al., 1974; Hess et. al., 1987), even though their contribution to the chemistry of light atoms is negligible. All calculated interaction energies were corrected for the basis set superposition error (BSSE) using the counterpoise correction (Boys et. al., 1970).

The presented relativistic MP2 and CCSD(T) calculations were performed in two different all-electron basis sets. The relativistic Atomic Natural Orbital basis sets (ANO-RCC) were contracted according to the recommended contraction, denoted as VDZP, VTZP or VQZP. The ANO-RCC basis sets include polarization (all contractions) and diffuse (VTZP and VQZP contractions) functions. The benchmark results (reference data for comparison purposes) were obtained using the aug-cc-pVXZ-DK (X = D, $\mathrm{T}, \mathrm{Q})$ basis sets, developed for relativistic calculations and well suited for the description of weakly interacting systems.

\section{Extrapolation schemes}

Three types of extrapolation techniques to obtain interaction energies in the complete basis set limit 
were used in this study. Herein, the interaction energies were obtained by 2-point extrapolation techniques. The first extrapolation technique (I) used was the one designed by Halkier et. al.:

$$
\Delta E_{C B S}^{c o r r}=\frac{Y^{3} \Delta E_{Y}^{c o r r}-X^{3} \Delta E_{Y}^{c o r r}}{Y^{3}-X^{3}}
$$

where $\Delta E_{X}^{c o r r}$ and $\Delta E_{Y}^{c o r r}$ represent correlation interaction energies calculated in the double- $\zeta$ and triple- $\zeta$ basis sets (DT extrapolation) or triple- $\zeta$ and quadruple- $\zeta$ basis sets (TQ extrapolation), respectively. Parameters $\mathrm{X}$ and $\mathrm{Y}$ correspond to the cardinality number 2, 3 and 4 in case of the double- $\zeta$, triple- $\zeta$ and quadruple- $\zeta$ basis set, respectively. The interaction energies were obtained by adding $\Delta E_{C B S}^{c o r r}$ to the HF interaction energy calculated in the higher cardinality number basis set used in the given extrapolation.

The second approach (II), designed by Truhlar, is based on the extrapolation of interaction energies calculated in the double- $\zeta$ and triple- $\zeta$ basis sets only. The complete basis set limit for the HartreeFock (HF) method was obtained as:

$$
\Delta E_{C B S}^{H F}=\frac{3^{\alpha}}{3^{\alpha}-2^{\alpha}} \Delta E_{T}^{H F}-\frac{2^{\alpha}}{3^{\alpha}-2^{\alpha}} \Delta E_{D}^{H F},
$$

where $\Delta E_{D}^{H F}$ and $\Delta E_{T}^{H F}$ correspond to energies computed with double- $\zeta(\mathrm{D})$ and triple- $\zeta(\mathrm{T})$ basis sets, respectively. A similar expression was used for correlation energy extrapolation:

$$
\Delta E_{C B S}^{c o r r}=\frac{3^{\beta}}{3^{\beta}-2^{\beta}} \Delta E_{T}^{c o r r}-\frac{2^{\beta}}{3^{\beta}-2^{\beta}} \Delta E_{D}^{c o r r},
$$

where $\alpha$ and $\beta$ are the extrapolation exponents optimized for HF and MP2 or CCSD(T) methods. Their values are 3.4 for $\alpha$ (HF in eq. 3), whereas $\beta$ in eq. 4 is equal to 2.2 for the MP2 and to 2.4 for the CCSD(T) method. The $\Delta E_{D}^{\text {corr }}$ and $\Delta E_{T}^{\text {corr }}$ are correlation interaction energies calculated in the double- $\zeta$ and triple- $\zeta$ basis sets. The interaction energy evaluated at the complete basis set limit is equal to the sum of the $\Delta E_{C B S}^{H F}$ and $\Delta E_{C B S}^{c o r r}$ energies.

The last extrapolation scheme (III) used is based on linear extrapolation. In this case, the energy depends on the number of functions of the basis set:

$$
\Delta E_{i}=\Delta E_{C B S}+a X_{i},
$$

where $X_{i}$ represents the reciprocal number of basis set functions, $\Delta E_{i}$ is the particular interaction energy calculated in the given basis set and $\triangle E_{C B S}$ is the extrapolated complete basis set limit interaction energy. The number of functions corresponds to the number of contracted functions. For example, the size of the contracted ANO-RCG-VQZP basis set for an $\mathrm{H}$ atom is $4 \mathrm{~s} 3 \mathrm{p} 2 \mathrm{~d} 1 \mathrm{f}$ which corresponds to 30 functions. Still, the complete basis set limit interaction energies (in eq. 5) can be obtained in two different ways, the first being based on the addition of the extrapolated MP2 or CCSD(T) correlation interaction energies evaluated by eq. (5) to the Hartree-Fock interaction energies calculated in the larger basis set used in the two point extrapolation, and the second being based on direct extrapolation of the total interaction energies, i. e. $\Delta E_{i}$ in eq. (5) represent the sum of HartreeFock and correlation interaction energies.

All three extrapolation techniques mentioned above (I-III) were applied to assess the complete basis set limit of the ANO-RCC basis sets. The interaction energies calculated in the aug-cc-pVXZ-DK basis sets were extrapolated by the Halkier's and Truhlar's approaches (I and II) and were considered as the reference interactions.

\section{Results}

The MP2/CBS interaction energies (Table 1) of the complexes studied were obtained by direct extrapolation [(eqs (2)-(5)], i. e. by extrapolation of MP2 interaction energies calculated with various contracted ANO-RCG and/or aug-cc-pVXZDK basis sets. While the Halkier's and Truhlar's approaches are based on the extrapolation of MP2 correlation interaction energies [Eqs. (2) and (4)], linear extrapolation with a number of basis set functions [eq. (5)] with correlation as well as total MP2 interaction energies. The CCSD(T)/CBS interaction energies (Table 2) were evaluated first by direct extrapolation techniques as it was the case for the MP2/CBS interaction energies. Moreover, the $\operatorname{CCSD}(\mathrm{T}) / \mathrm{CBS}$ interaction energies were obtained also in the indirect way (Table 3) using eq. (1). This method is based on the addition of the difference between CCSD(T) and MP2 interaction energies calculated the smaller basis set to the MP2/CBS interaction energy.

Extrapolation schemes used are labeled as follows: e.g. label Halkier/Truhlar [TQ] corresponds to the direct Halkier/Truhlar extrapolation with contracted triple- $\zeta$ and quadruple- $\zeta$ basis sets; indirect CCSD(T)/CBS interaction energy extrapolations are showed by square brackets containing the contractions of basis sets used for MP2/CBS as well as the basis set contraction used for the evaluation of the difference between $\operatorname{CCSD}(\mathrm{T})$ and MP2 interaction energies calculated in the smaller basis set (label 1/n corr[DT-D] corresponds to the use of linear extrapolation of MP2 correlation energies calculated in contracted double- $\zeta$ and triple- $\zeta$ basis sets, and the difference between $\operatorname{CCSD}(\mathrm{T})$ and MP2 interaction energies was calculated in double- $\zeta$ basis set). Note that all Tables presented contain the basis set 
limit interaction energies calculated at the aug-ccpVXZ-DK as well as for the ANO-RCG basis sets. As mentioned earlier, the aug-cc-pVXZ-DK results were used as reference data in this study.

Table 1 shows that the MP2/CBS interaction energies calculated in ANO-RCG basis sets agree well with the reference aug-cc-pVXZ-DK data. The worst agreement (about $0.1 \mathrm{kcal} / \mathrm{mol}$ ) with the reference data was obtained for extrapolations with the ANO-RCG-VDZP and ANO-RCG-VTZP basis sets. However, it is necessary to note that the Truhlar extrapolation exponents are not tailored for ANORCG basis sets. Moreover, the ANO-RCC-VDZP basis set is rather small and contains only the polarization function. The absence of the diffuse functions in the ANO-RCC-VDZP basis set seems to be crucial for the assessment of interaction energies in noncovalent complexes. However, the MP2/CBS interaction energies obtained from the ANO-RCG-VTZP and ANO-RCC-VQZP basis sets provide very good agreement with the benchmark data, especially in case of the Halkier's extrapolation. However, description of the benzene...methane complex at the MP2 level with ANO-RCC basis sets is poorer. The best results were obtained using the Halkier's [DT] and [TQ] extrapolations. The use of other extrapolation techniques for the description of this complex led to an overestimation of the interaction energies in comparison with reference data, although these differences are actually small (about $0.2 \mathrm{kcal} / \mathrm{mol}$ ).

The CCSD(T)/CBS interaction energies obtained by direct extrapolation with ANO-RCG-VDZP and ANO-RCG-VTZP basis sets as compiled in Table 2 are in poor agreement with the reference data
(Halkier's [TQ] extrapolation of the aug-cc-pVXZDK basis sets). However, Halkier's [TQ] extrapolation provides accurate results which are comparable with the benchmark results. On the other hand, extrapolation of $1 / \mathrm{n}$ corr and $1 / \mathrm{n}$ total of ANORCG basis sets overestimate the interaction energies. However, deviations from reference results are small (about $0.2 \mathrm{kcal} / \mathrm{mol}$ ), with the highest values found for the HF...methanol complex.

The CCSD(T)/CBS interaction energies calculated using indirect extrapolation as compiled in Table 3 provide results corresponding to those of the direct techniques. Also indirect extrapolation with the ANO-RGG-VDZP and ANO-RGG-VTZP basis sets combination is insufficient. The reason for the lower extrapolation accuracy is the absence of diffuse functions in the ANO-RCG-VDZP basis set. Interestingly, interaction energies of the benzene... methane complex extrapolated with the ANORCG-VDZP and ANO-RCG-VTZP basis sets are overestimated. The ANO-RCG-VTZP and ANORCC-VQZP basis sets used for the extrapolation to the complete basis set limit provide results well comparable with the benchmark reference data.

\section{Conclusions}

Results obtained with the ANO-RCC basis sets are comparable with the benchmark aug-cc-pVXZ-DK data. However, the calculations have to be performed with the ANO-RCC-VTZP and higher basis sets. The use of the ANO-RCC-VDZP basis set provides poorer estimation of the interaction energies at the complete basis set limit. These poorer $( \pm 0.5 \mathrm{kcal} / \mathrm{mol})$ results

Tab. 1. DKH BSSE corrected MP2/CBS interaction energy $\Delta \mathrm{E}[\mathrm{kcal} / \mathrm{mol}]$ of studied complexes. $\Delta \mathrm{E}$ was obtained by direct Halkier's [eq. (2)] and Truhlar's [eq. (3) and (eq. (4)] as well as by linear extrapolation of correlation or total interaction energies [eq. (5)]. Basis sets used in extrapolations are presented in square parentheses. Results obtained by Halkier's [TQ] extrapolation of aug-ccpVXZ-DK basis sets represent the reference data.

\begin{tabular}{|c|c|c|c|c|c|}
\hline & methane... $\mathrm{F}_{2}$ & water...ammonia & water...water & HF...methanol & benzene...methane \\
\hline \multicolumn{6}{|l|}{ ANO-RCC } \\
\hline Halkier [DT] & -0.442 & -6.358 & -4.669 & -9.091 & -1.823 \\
\hline Halkier [TQ] & -0.543 & -6.573 & -4.982 & -9.594 & -1.857 \\
\hline Truhlar [DT] & -0.478 & -6.291 & -4.760 & -9.083 & -2.014 \\
\hline $1 / \mathrm{n} \operatorname{corr}[\mathrm{DT}]$ & -0.495 & -6.457 & -4.751 & -9.161 & -2.042 \\
\hline $1 / \mathrm{n} \operatorname{corr}[\mathrm{TQ}]$ & -0.573 & -6.633 & -5.046 & -9.688 & -1.920 \\
\hline 1/n total $[\mathrm{DT}]$ & -0.474 & -6.114 & -4.777 & -9.007 & -2.039 \\
\hline 1/n total [TQ] & -0.578 & -6.617 & -5.095 & -9.751 & -1.902 \\
\hline \multicolumn{6}{|c|}{ aug-cc-pVXZ-DK } \\
\hline Halkier [DT] & -0.541 & -6.483 & -4.853 & -9.389 & -1.801 \\
\hline Halkier [TQ] & -0.548 & -6.583 & -4.973 & -9.610 & -1.815 \\
\hline Truhlar [DT] & -0.573 & -6.617 & -4.939 & -9.585 & -1.867 \\
\hline
\end{tabular}


can be explained by the absence of diffuse functions in the ANO-RCC-VDZP basis set.

Still, ANO-RCG basis sets proved to be useful for obtaining interaction energies at the complete basis set limit while providing an order of magnitude better results for the studied noncovalent systems with respect to chemical accuracy. Moreover, the all electron ANO-RCG basis sets are available for the

Tab. 2. DKH BSSE corrected CCSD(T)/CBS interaction energy $\Delta \mathrm{E}[\mathrm{kcal} / \mathrm{mol}]$ of studied complexes. $\Delta \mathrm{E}$ was obtained by direct Halkier's [eq. (2)] and Truhlar's [eq. (3) and (eq. (4)] extrapolations as well as by linear extrapolation of correlation or total interaction energies [eq. (5)]. Basis sets used in extrapolations are presented in square parentheses. Results obtained by Halkier's [TQ] extrapolation of aug-cc-pVXZ-DK basis sets represent the reference data. High demands of the CCSD(T) method do not allow performing calculations of some studied systems in large aug-cc-pVTZ-DK or aug-cc-pVQZ-DK.

\begin{tabular}{|c|c|c|c|c|c|}
\hline & methane $\ldots \mathrm{F}_{2}$ & water...ammonia & water...water & HF...methanol & benzene...methane \\
\hline \multicolumn{6}{|l|}{ ANO-RCC } \\
\hline Halkier $[\mathrm{DT}]$ & -0.382 & -6.358 & -4.744 & -9.152 & -1.443 \\
\hline Halkier [TQ] & -0.499 & -6.547 & -5.057 & -9.650 & \\
\hline Truhlar [DT] & -0.409 & -6.315 & -4.852 & -9.195 & -1.574 \\
\hline 1/n corr $[\mathrm{DT}]$ & -0.444 & -6.537 & -4.890 & -9.336 & -1.664 \\
\hline $1 / \mathrm{n} \operatorname{corr}[\mathrm{TQ}]$ & -0.534 & -6.623 & -5.137 & -9.772 & \\
\hline 1/n total $[\mathrm{DT}]$ & -0.424 & -6.194 & -4.916 & -9.182 & -1.661 \\
\hline 1/n total [TQ] & -0.540 & -6.606 & -5.187 & -9.835 & \\
\hline \multicolumn{6}{|c|}{ aug-cc-pVXZ-DK } \\
\hline Halkier [DT] & -0.502 & -6.459 & -4.919 & -9.418 & \\
\hline Halkier [TQ] & & -6.527 & -5.026 & & \\
\hline Truhlar [DT] & -0.527 & -6.572 & -4.989 & -9.596 & \\
\hline
\end{tabular}

Tab. 3. DKH BSSE corrected CCSD $(\mathrm{T}) / \mathrm{CBS}$ interaction energy $\Delta \mathrm{E}[\mathrm{kcal} / \mathrm{mol}]$ of studied complexes. CCSD(T)/CBS interaction energies were obtained by indirect extrapolation described by eq. (1). MP2/CBS interaction energies were evaluated by Halkier's [eq. (2)] and Truhlar's [eq. (3) and (eq. (4)] as well as by linear extrapolation of correlation or total interaction energies [eq. (5)]. Basis sets used in extrapolations are showed in square parentheses. Results obtained by Halkier's [TQ-T] or [TQ-D] extrapolation of aug-cc-pVXZ-DK basis sets represent the reference data, respectively. High demands of the CCSD(T) method do not allow performing calculation of some studied systems in large aug-cc-pVTZ-DK or aug-cc-pVQZ-DK.

\begin{tabular}{|c|c|c|c|c|c|}
\hline & methane... $\mathrm{F}_{2}$ & water...ammonia & water...water & HF...methanol & benzene...methane \\
\hline \multicolumn{6}{|l|}{ ANO-RCC } \\
\hline Halkier $[\mathrm{DT}+\mathrm{D}]$ & -0.341 & -5.934 & -4.424 & -8.613 & -1.430 \\
\hline Halkier $\left[\mathrm{TQ}^{+} \mathrm{D}\right]$ & -0.441 & -6.149 & -4.737 & -9.115 & -1.464 \\
\hline Halkier $\left[\mathrm{TQ}^{+} \mathrm{T}\right]$ & -0.470 & -6.447 & -4.962 & -9.495 & -1.473 \\
\hline Truhlar $[\mathrm{DT}+\mathrm{D}]$ & -0.376 & -5.867 & -4.516 & -8.605 & -1.621 \\
\hline $1 / \mathrm{n} \operatorname{corr}[\mathrm{DT}+\mathrm{D}]$ & -0.393 & -6.033 & -4.506 & -8.683 & -1.649 \\
\hline $1 / \mathrm{n} \operatorname{corr}\left[\mathrm{TQ}^{+} \mathrm{D}\right]$ & -0.471 & -6.209 & -4.801 & -9.210 & -1.527 \\
\hline $1 / \mathrm{n} \operatorname{corr}\left[\mathrm{TQ}^{+\mathrm{T}}\right]$ & -0.501 & -6.508 & -5.026 & -9.589 & -1.536 \\
\hline 1/n total $[\mathrm{DT}+\mathrm{D}]$ & -0.373 & -5.690 & -4.533 & -8.529 & -1.646 \\
\hline 1/n total $\left[\mathrm{TQ}^{+} \mathrm{D}\right]$ & -0.477 & -6.193 & -4.851 & -9.273 & -1.509 \\
\hline $1 / \mathrm{n}$ total $[\mathrm{TQ}+\mathrm{T}]$ & -0.506 & -6.491 & -5.076 & -9.652 & -1.518 \\
\hline \multicolumn{6}{|l|}{ aug-cc-pVXZ-DK } \\
\hline Halkier $[\mathrm{DT}+\mathrm{D}]$ & -0.482 & -6.323 & -4.816 & -9.240 & -1.453 \\
\hline Halkier $[\mathrm{TQ}+\mathrm{D}]$ & -0.489 & -6.422 & -4.935 & -9.461 & -1.467 \\
\hline Halkier $\left[\mathrm{TQ}^{+} \mathrm{T}\right]$ & -0.503 & -6.518 & -5.008 & -9.587 & \\
\hline Truhlar $[\mathrm{DT}+\mathrm{D}]$ & -0.514 & -6.457 & -4.901 & -9.437 & -1.518 \\
\hline
\end{tabular}


entire periodic table. Although, the aug-cc-pVXZDK basis sets represent the best choice because of the presence of better tailored diffuse functions, the use of the ANO-RCG basis sets represents a competitive alternative in noncovalent systems research.

\section{Acknowledgment}

This work was supported by the Slovak Grant Agency VEGA under the Contract No. 1/0598/16. We are grateful to the HPC Center at the Slovak University of Technology in Bratislava, which is a part of the Slovak Infrastructure of High Performance Computing (SIVVP project, ITMS code 26230120002, funded by the European Region Development Funds, ERDF) for the computational time and resources provided.

\section{References}

Berka K, Laskowski R, Riley KE, Hobza P, Vondrášek J (2009) J. Chem. Theory Comput. 5: 982-992.

Boys SF, Bernardi F (1970) Mol. Phys. 19: 553.

Douglas M, Kroll NM (1974) Ann. Phys. 82: 89.

Granatier J, Dubecký M, Lazar P, Otyepka M, Hobza P (2013) J. Chem. Theory Comput. 9: 1461-1468.

Granatier J, Lazar P, Otyepka M, Hobza P (2011) Chem. Theory Comput. 7: 3743-3755.

Granatier J, Urban M, Sadlej AJ (2010) Chem. Phys. Lett. 484: 154-159.

Halkier A, Helgaker T, Jorgensen P, Klopper W, Koch H, Olson J, Wilson AK (1998) Chem. Phys. Lett. 286: 243.

Hess BA, Chandra P (1987) Phys. Scr. 36: 412.

Jurečka P, Hobza P (2002) Chem. Phys. Lett. 365: 89-94.

Jurečka P, Šponer J, Černý J, Hobza P (2006) Phys. Chem. Chem. Phys. 8: 1985-1993.
Karlström G, Lindh R, Malmqvist PÅ, Roos BO, Ryde U, Veryazov V, Widmark PO, Cossi M, Schimmelpfennig B, Neogrády P, Seijo L (2003) Comput. Mater. Sci. 28: 222.

Martin JML (1996) Chem. Phys. Lett. 259: 669.

Martin JML, Taylor PR (1997) J. Chem. Phys. 105: 8620.

Martin JML (1998) Am. Chem. Soc. Symp. Ser. 677: 212.

Møller C, Plesset MS (1934) Phys. Rev. 46: 0618.

Peterson KA (2015) J. Chem. Phys. 142: 074105.

Pitoňák M, Heßelmann AJ (2010) Chem. Theory Comput. 6: 168.

Pitoňák M, Janowski T, Neogrády P, Pulay $\mathrm{P}$, Hobza $\mathrm{P}$ (2009) J. Chem. Theory Comput. 5: 1761.

Pitoňák M, Riley KE, Neogrády P, Hobza P (2008) Chem. Phys. Chem. 9: 1636.

Raghavachari K, Trucks G, Pople J, Head-Gordon M (1989) Chem Phys. Lett. 157: 479.

Riley KE, Merz KM (2006) J. Phys. Chem. B 110 (32): 15650-15653.

Riley KE, Pitoňák M, Jurečka P, Hobza P (2010). Chem. Rev. 110: 5023

Roos BO, Lindh R, Malmqvist PA, Veryazov V, Widmark PO (2004) J. Phys. Chem. A 108: 2851.

Roos BO, Lindh R, Malmqvist PA, Veryazov V, Widmark PO (2005) J. Phys. Chem. A 109: 6575.

Řezáč J, Hobza P (2013) J. Chem. Theory Comput. 8: 2151-2155.

Řezáč J, Riley KE, Hobza P (2011) J. Chem. Theory Comput. 7: 2427-2438.

Řzáč J, Riley KE, Hobza P (2012) J. Chem. Theory Comput. 8: 4285-4292.

Truhlar DG (1998) Chem. Phys. Lett. 294: 45-48.

Vondrášek J, Bendová L, Klusák V, Hobza P (2005) J. Am. Chem. Soc. 127: 2615-2619.

Vondrášek J, Kubař T, Jenney FE, Adams MWW, Kožíšek M, Černý J, Sklenář V, Hobza P (2007) Chem.-Eur. J. 13: 9022-9027. 